\title{
OCULAR AND ORBITAL METASTASIS IN BREAST CANCER: CASE SERIES REPORT
}

Thaís R. Gontijo', Nilceana M. A. Freitas', Ana F. P. G. Campedelli', Nathalya A. Yagi', Sylvia S. Pires', Mattheus H. Vale' ${ }^{1}$ Hospital Araújo Jorge, Centro Brasileiro de Radioterapia, Oncologia e Mastologia -Goiânia (GO), Brazil.

Objectives: Breast cancer is the most prevalent cancer subtype among women and it is also the leading cause of cancer death in women. It can spread disease to all kind of structures, including to the orbit and eye, also being responsible for $2-7 \%$ of metastasis. Most patients presenting with ocular metastases already have a history of treated primary cancer. The majority of ocular and orbital metastasis is a primary result of breast (47\%), lung (21\%), and gastrointestinal tract (4\%) lesions. The aim of this report is to analyze the efficacy of radiotherapy treatment in ocular and orbital metastasis from breast cancer in women. Methods: It is a descriptive study of four case reports of ocular and orbital metastasis in breast cancer patients, during a year from 2017 to 2018, who were treated in a public hospital and a private clinic. Case report: Among the four case reports, only one patient had a newly diagnosed cancer by its visual symptoms as the first presentation of breast cancer; in the others, disease-free survival in breast cancer patients was five to eight years, and, in one of them, the ocular metastasis occurred simultaneously with multiple metastasis in nervous system. After beginning treatment, all the symptoms initially reported, such as diplopia, blurry vision, decrease in visual acuity, eye pain, inflammation and redness of the eye, conjunctival injection, loss of eye motility, and palpebral ptosis were softened. Excluding one of them, all the four patients received radiation therapy in the orbit. Regarding the case with the newly diagnosed patient, it was treated with hormone therapy (Fulvestrant) and did not undergo radiotherapy. For other patient, it was given intensitymodulated radiation therapy (IMRT) with complete treatment response after 3 months treatment. For the third patient, brain tridimensional radiation therapy (RT3D) was preferred due to the presence of other metastasis in the nervous system. This patient had her visual symptoms minimized, but still presented other neurological disorders because of multiples brain metastasis. The fourth patient is still waiting for the beginning of radiation therapy. Conclusion: Despite rare, ocular and orbital metastasis is strongly correlated to patients with breast cancer history and radiation therapy can be an important therapeutic arsenal to local control. 